\title{
Graft Copolymers from Poly(vinylidene fluoride-co- chlorotrifluoroethylene) via Atom Transfer Radical Polymerization
}

\author{
Mingfu Zhang*, Thomas P. Russell ${ }^{*}$ \\ Department of Polymer Science and Engineering, University of Massachusetts, \\ Amherst, MA 01003, USA
}

\section{Supporting information}

\section{Mass spectroscopy measurement of poly(chlorotrifluoroethylene) (PCTFE) oligomer}

The mass spectrum was acquired in FAB ionization mode on a JMS-700 MStation double focusing mass spectrometer (JEOL Inc, Peabody MA). 1 mg of sample was suspended in 3-nitrobenzyl alcohol and ionization was achieved by Xe atoms accelerated to $30 \mathrm{keV}$.

The mass spectrum is shown in Figure A. It is clear that the PCTFE oligomer, which was used as the initiator in the model reactions, is a mixture of PCTFE chains with different chain lengths. It contains some species with molecular weight higher than 1000, although the content of these high molecular weight species may be quite low. Because each CTFE unit carries one initiating site, the molecular weight distribution (MWD) of the PCTFE oligomer has dramatic influence on the MWD of the resultant polymers.

\section{${ }^{13}$ C-NMR measurement of poly(chlorotrifluoroethylene) (PCTFE) oligomer}

${ }^{13} \mathrm{C}$ - NMR measurement was performed on a Bruker DPX 300 spectrometer without ${ }^{19} \mathrm{~F}$ decoupling. $\mathrm{CDCl}_{3}$ was used as solvent. The ${ }^{13} \mathrm{C}$-NMR spectrum is shown in Figure B. Due to the strong coupling between ${ }^{13} \mathrm{C}$ and ${ }^{19} \mathrm{~F}$, each ${ }^{13} \mathrm{C}$ peak was split into multi peaks. The peak locations agree fairly well with those reported in literature, ${ }^{1}$ but it is difficult to interpret all the peaks in Figure B because of the influence of end groups and the possibility of different bonding arrangements (head-to-tail and head-to-head). Thus it 
is hard to get the clear information about the end group from the ${ }^{13} \mathrm{C}-\mathrm{NMR}$ measurement. From the elemental analysis, the chlorine content in the PCTFE oligomer is $38.8 \mathrm{w} \%$, which is considerably higher than that in the CTFE unit (30.44 w\%). Thus most probably the end groups of the PCTFE oligomer contain chlorine.

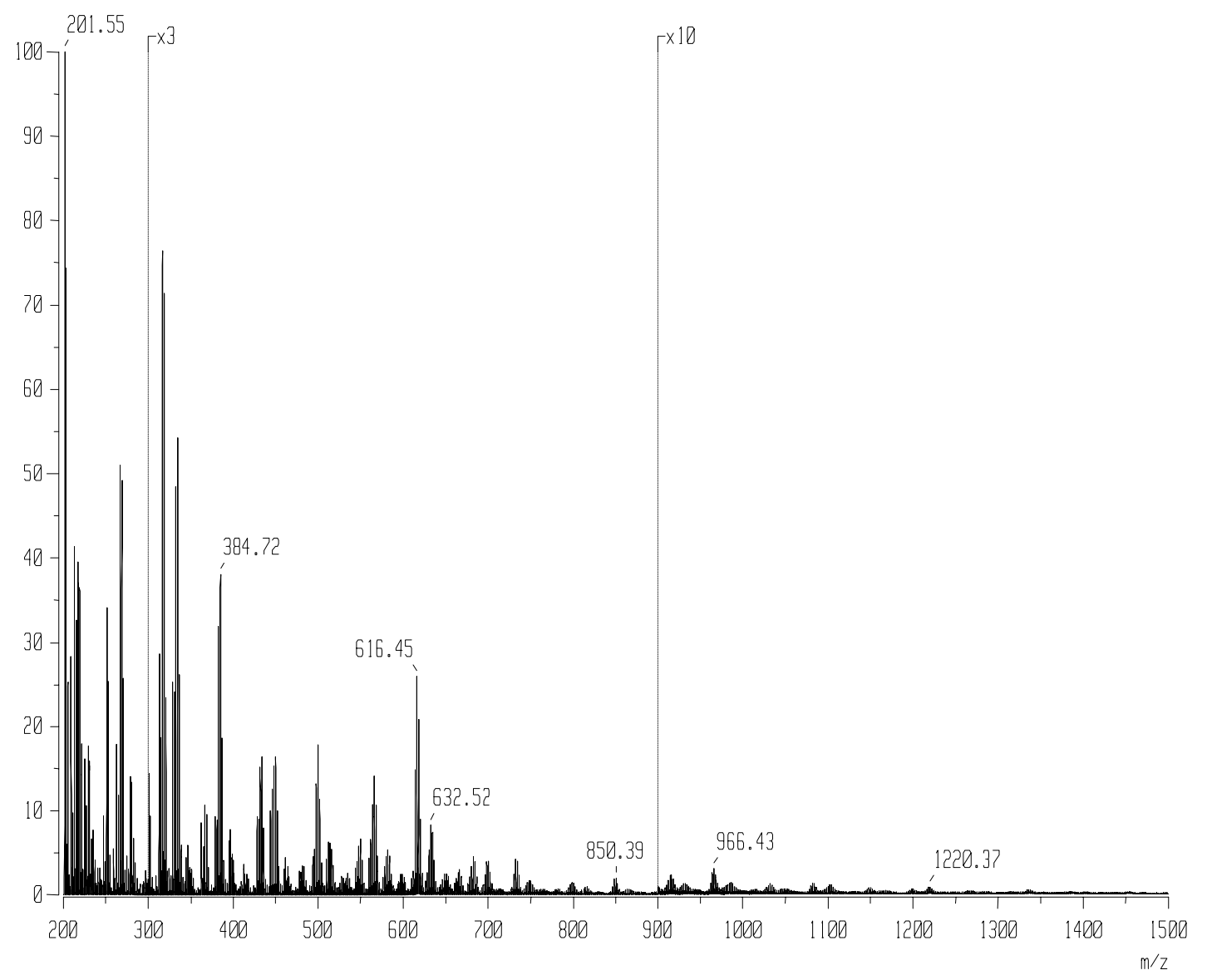

Figure A. Mass spectrum of PCTFE oligomer (Ion mode: FAB+; Spectrum type: normal ion (MF-linear)); Note that the intensity between $300-900 \mathrm{~m} / \mathrm{z}$ was magnified by a factor of 3 and the intensity above $900 \mathrm{~m} / \mathrm{z}$ was magnified by a factor of 10 . 


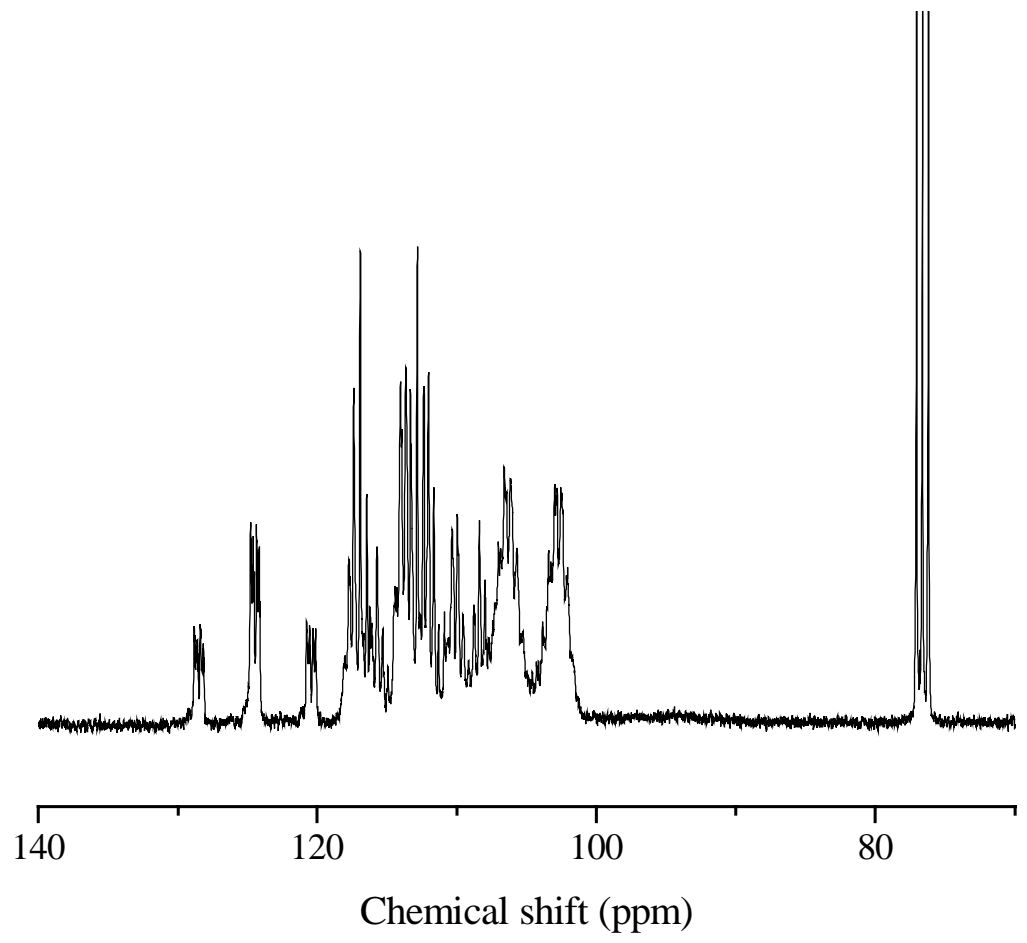

Figure B. ${ }^{13} \mathrm{C}$-NMR spectrum of the PCTFE oligomer in $\mathrm{CDCl}_{3}$ (The triple peak located at $\sim 77 \mathrm{ppm}$ corresponds to the solvent $\left(\mathrm{CDCl}_{3}\right)$ peak).

\section{Reference:}

(1) Ishida, T.; Suzuki, T.; Takimoto, Y.; Sasakura, H.; Jitsugiri, Y. Surface Coatings International, Part B: Coatings Transactions 2002, 85, 143-148. 